\title{
Apport de la sédation intraveineuse sous midazolam en chirurgie buccale: étude clinique menée à l'Ortenau Kilinikum de Kiehl (Allemagne)
}

\author{
Aurélie Scheer, Pierre Keller \\ Faculté de Chirurgie dentaire, 1 porte de l'Hôpital, 67000 Strasbourg, France \\ aurélie.scheer@gmail.com
}

La sédation consciente se définit comme étant la diminution ou l'abolition des réponses physiologiques et psychologiques face à une situation stressante, sans perte de conscience, de coopération et des réflexes de protection; la respiration spontanée et les reflexes sont donc maintenus. Cet effet est obtenu aux moyens de techniques et d'agents qui induisent un stade de dépression du système nerveux central avec une marge de sécurité suffisante pour rendre la perte de conscience impossible à atteindre. Elle permet des traitements chirurgicaux sans stress psychiques et physiologiques pour le patient et en améliorant sa coopération, en particulier pour des opérations longues et compliquées.

A l'heure actuelle, la sédation par inhalation de mélange équimolaire d'oxygène et de protoxyde d'azote est l'une des méthodes de sédation consciente les plus utilisées en France (Wagner 2011). Cette technique présente néanmoins des limites chez les patients très angoissés ou lors des interventions de chirurgie orale nécessitant une longue coopération du patient.

Les benzodiazépines ont des propriétés anxiolytiques, anticonvulsivantes, sédatives, amnésiantes, myorelaxantes et hypnotiques (Calderon 2009). Le midazolam est une benzodiazépine avec un temps de demi-vie très court. Il est largement utilisé en réanimation à des fins sédatives, pour ses propriétés anxiolytiques et amnésiantes. La bonne tolérance hémodynamique de cette molécule et l'existence d'un antagoniste (flumazénil) rendent son utilisation sûre (Pourriat 2002). Cependant, ses effets montrent une grande variabilité interindividuelle qui nécessite une adaptation de la posologie. Avec l'administration intraveineuse, contrairement à l'administration orale, rectale ou intra-musculaire, la

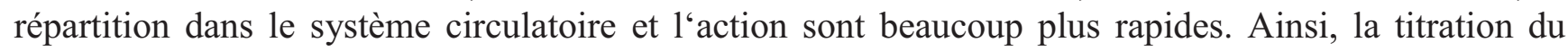
midazolam permet de modifier la profondeur de la sédation de chaque patient et ce durant toute la durée de l'intervention. Les propriétés pharmacodynamiques du midazolam, administré par voie intraveineuse, lui permettent donc de trouver son intérêt lors d'interventions longues et traumatisantes, et dans les cas de patients peu coopérants ou phobiques (Gotkay et al. 2001). En France, son utilisation est réservée au milieu hospitalier. Cependant en Allemagne, une formation et un équipement adapté autorise le chirurgien-dentiste à utiliser cette technique dans son cabinet.

L'objectif de cette étude est d'évaluer la satisfaction des patients pris en charge au moyen de cette technique de sédation intraveineuse. 